\title{
A Design of New Airborne Integrated Alarm System
}

\author{
Jianhua Li", Jintao Xie, Xun Li, Chaogang Hu \\ Jiangxi Hongdu Aviation Industry Group Co., Ltd, Nanchang, China \\ Email address: \\ 774778019@qq.com (Jianhua Li),dz52mm@163.com (Jianhua Li) \\ ${ }^{*}$ Corresponding author
}

\section{To cite this article:}

Jianhua Li, Jintao Xie, Xun Li, Chaogang Hu. A Design of New Airborne Integrated Alarm System. Journal of Electrical and Electronic Engineering. Vol. 7, No. 2, 2019, pp. 64-68. doi: 10.11648/j.jeee.20190702.16

Received: March 12, 2019; Accepted: May 27, 2019; Published: June 15, 2019

\begin{abstract}
Aiming at the fault alarm requirement of a certain type of aircraft, a new type of airborne integrated alarm system is designed. The system adopts modular design idea, and the acquisition part is composed of multi-slice shift register 54HC165 cascade, which can reduce the number of control pins; the main control part uses the heterogeneous processing scheme of DSP and FPGA, which not only ensures the real-time processing of information, but also enriches the interactive ability of the interface; the output part uses a single FPGA to control the alarm lamp box, giving full play to the system advantages of centralized alarm and hierarchical processing. potential. Ground debugging experiments show that the system can accurately judge the aircraft warning information, and quickly notify the pilot according to the degree of emergency. The scheme is simple to implement, and the interface is scalable, so it has high practical value with strong portability and expansibility.
\end{abstract}

Keywords: Airborne, Alarm System, Modular Design, Hierarchical Process

\section{Introduction}

With the rapid development of aviation technology, internal sensors and electronic equipment on the aircraft are becoming more and more complex, the pilot needs to face massive flight data when performing the corresponding tasks. [1] Once some abnormal information is omitted, serious accidents may occur. So a kind of specific system is urgently needed in the cockpit of aircraft to assist the pilot to receive status information of each airborne system, when the aircraft fall into dangerous situation, the pilot can be alerted in time to ensure flight safety [2].

In the common aircraft warning system, the warning system is very important for the evaluation of the health of the aircraft. Nowadays, the common alarm system mostly adopts the control system based on single chip microcomputer. With the increase of sensors for monitoring the flight status of aircraft, the monitored aircraft state parameters have become more and more complicated [3]. Traditional MCU processing systems will face difficulties in multitasking management and peripherals. This article is proposed in this context [4].

This paper designed an airplane integrated alarm system based on FPGA and DSP, the system analyzes the fault information of each system on the aircraft, and alarms in the form of light or voice, so that the pilot can accurately and quickly find out the operating status of the airplane, and take timely measures to reduce the probability of accidents [5].

\section{System Design}

The integrated alarm system consists of the following four parts: acquisition module, control module, output module and alarming lamp box, the design idea is shown in Figure 1. The status data that the system needs to process comes from two places, one is the discrete signal read by the acquisition module, theother is the RS-422A data packet sent by an electromechanical management computer. The control module obtains the alarm of each airborne subsystem by analyzing the above two parts of information, which plays an artificial voice on the inner communication box according to priority. The alarm information can be sent to the output module and airborne multi-function display via 422 channels. The output module controls the alarming lamp box in a specific way, and prompts the pilot to pay attention to the current abnormal condition with the light blinked. 


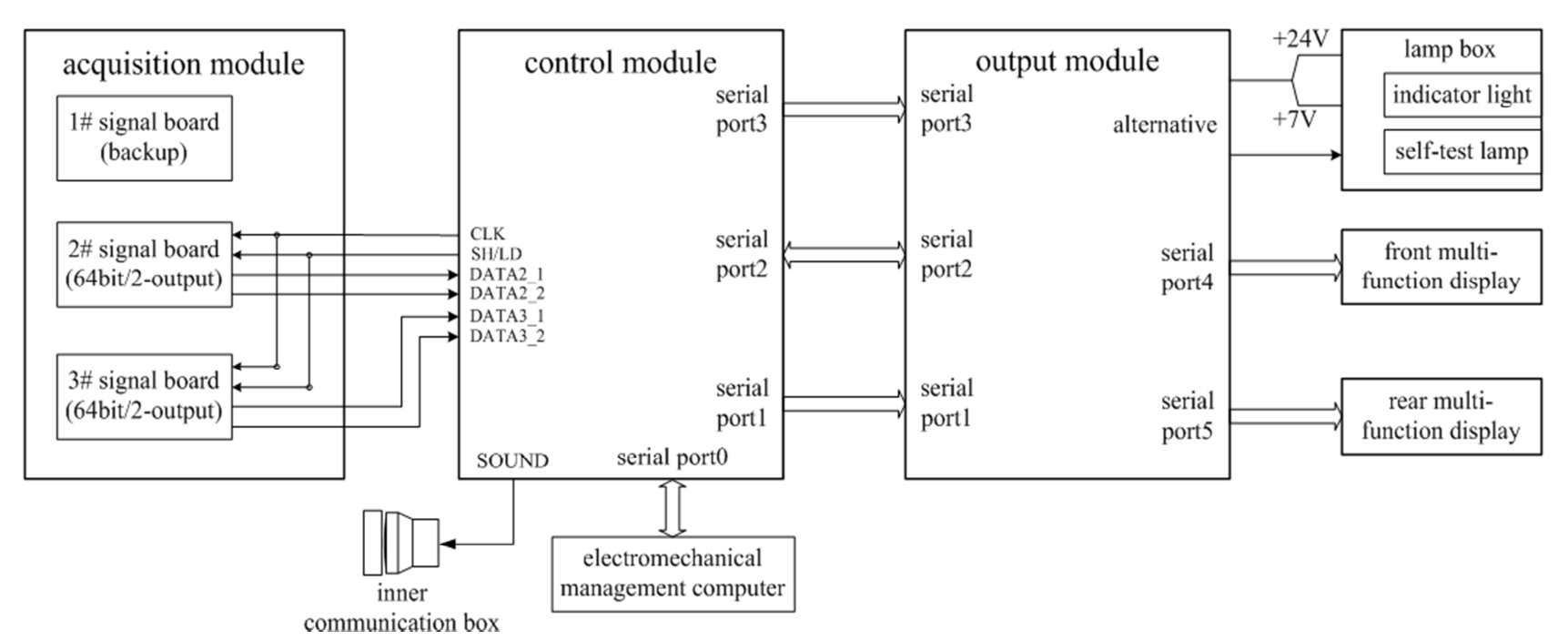

Figure 1. System design block diagram.

\section{Hardware Design}

\subsection{Acquisition Module Design}

The acquisition module is the input of the integrated alarm system. It consists of three signal boards, two of which work and one for backup, and is responsible for reading the discrete signals of the airborne equipment. The signal board is designed with $54 \mathrm{HC} 165$ chip [6]. The $54 \mathrm{HC} 165$ is an 8-bit high speed shift register. When the enable signal $\mathrm{SH} / \mathrm{LD}$ is valid, the parallel data $\mathrm{A} \sim \mathrm{F}$ is output bit by bit from the $\mathrm{QH}$ terminal under the control of the clock CLK, each acquisition cycle takes 32 clock cycles. All signal boards of the acquisition module share a set of clock and enable signal, but each retains two independent data channels DATA_1 and DATA_2. Each data channel is composed of four 54 shift register cascading, which can realize serial acquisition of 32 discrete signals, the schematic diagram is shown in Figure 2. The control module controls CLK and SH/LD to read all 128-bit discrete signals of the acquisition module through the DATA2_1, DATA2_2, DATA3_1 and DATA3_2, which greatly saves pin resources.

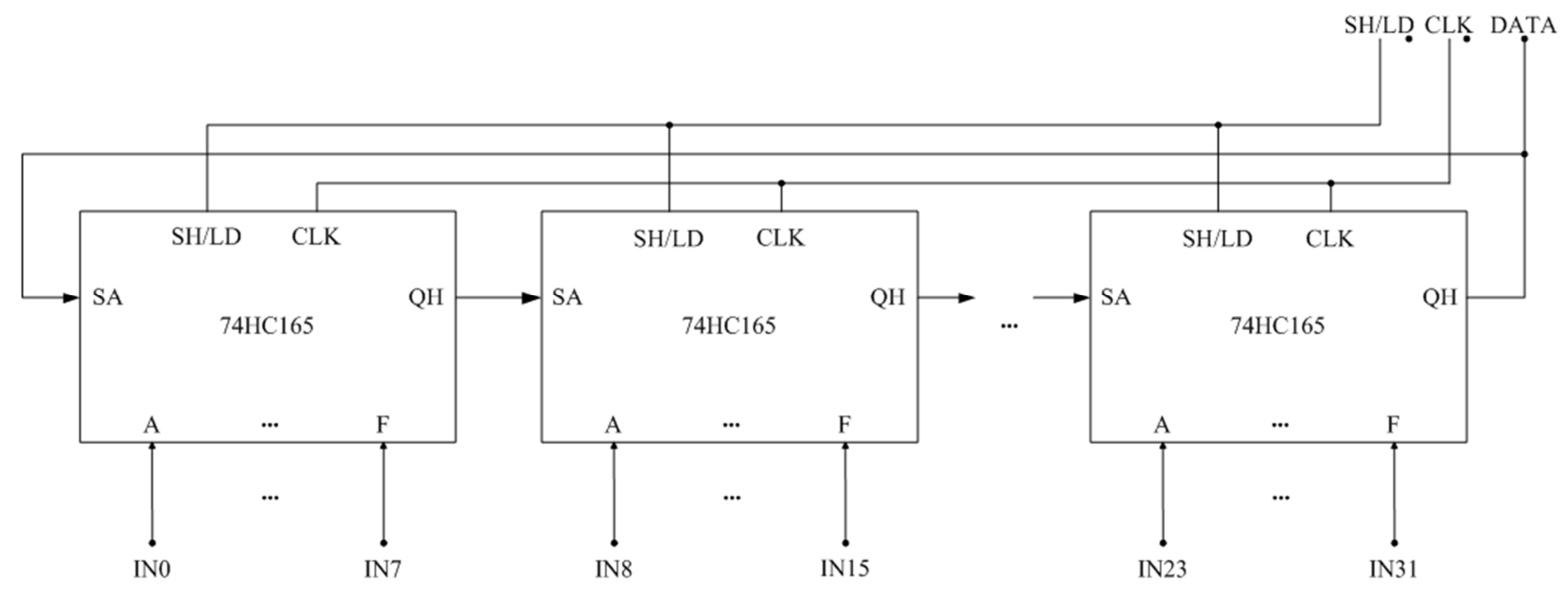

Figure 2. The schematic diagram of acquisition module.

\subsection{Control Module Design}

The control module is the core of the integrated alarm system. It consists of a DSP processor and an FPGA processor. It is responsible for the process scheduling of the entire system, the schematic diagram is shown in Figure 3 [7]. The DSP judges the alarm information, and the FPGA reads and converts the external data, the two exchange information through 16-bit XINTF bus with frequency of $67.5 \mathrm{MHz}$. The system has a total of 64 alarm voices, all stored in the DSP expansion 16M Flash. When DSP obtains the external information from the FPGA, it first judges based on multi-redundancy technology, removes the false condition, generates alarm information and sends it to the output Module; then search for the corresponding data from Flash, and write to VS1053 chip through SPI interface. VS1053 is a MP3 audio decoder with 2KB memory buffer, which converts MP3 data sent by DSP into alarm voice playing on the inner communication box. 


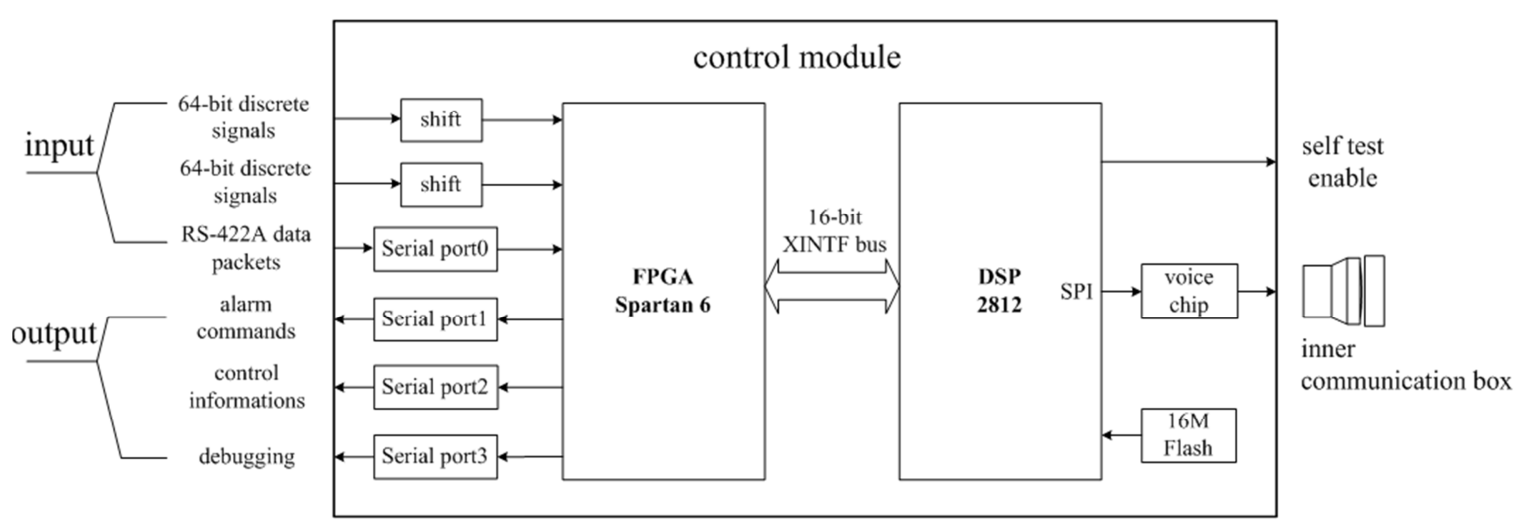

Figure 3. The schematic diagram of control module.

\subsection{Output Module Design}

The output module is the output of the integrated alarm system. It consists of an FPGA processor and several RS422 transceiver chips [8]. It is responsible for the control of alarming lamp box and the real-time transmission of alarm commands, the schematic diagram is shown in Figure 4. There are two kinds of 422 data packet sent by the control module. One is the alarm information for other systems, which is forwarded by RS422 transceiver chip to realize the synchronous output of 3-way instructions; The other is the control information for the alarming lamp box, which is analyzed by FPGA processor to realize three states switching of 11-way indicator light: on, off, and blinking. According to the design requirements, the brightness of alarming lamp box has two levels: day and night, the luminance in the day state is $510 \mathrm{~cd} / \mathrm{m}^{2}$, and the luminance in the night state is $25 \mathrm{~cd} / \mathrm{m}^{2}$. In order to achieve this function, the output module is designed with $24 \mathrm{~V} / 7 \mathrm{~V}$ voltage converting circuits which is controlled by FPGA processor. When the aircraft is in day state, the $24 \mathrm{~V}$ power output is enabled, when in night state, the $7 \mathrm{~V}$ power output is enabled, It realizes the brightness of alarming lamp display in two states: light-dark, which is convenient for pilots to use in day or night.

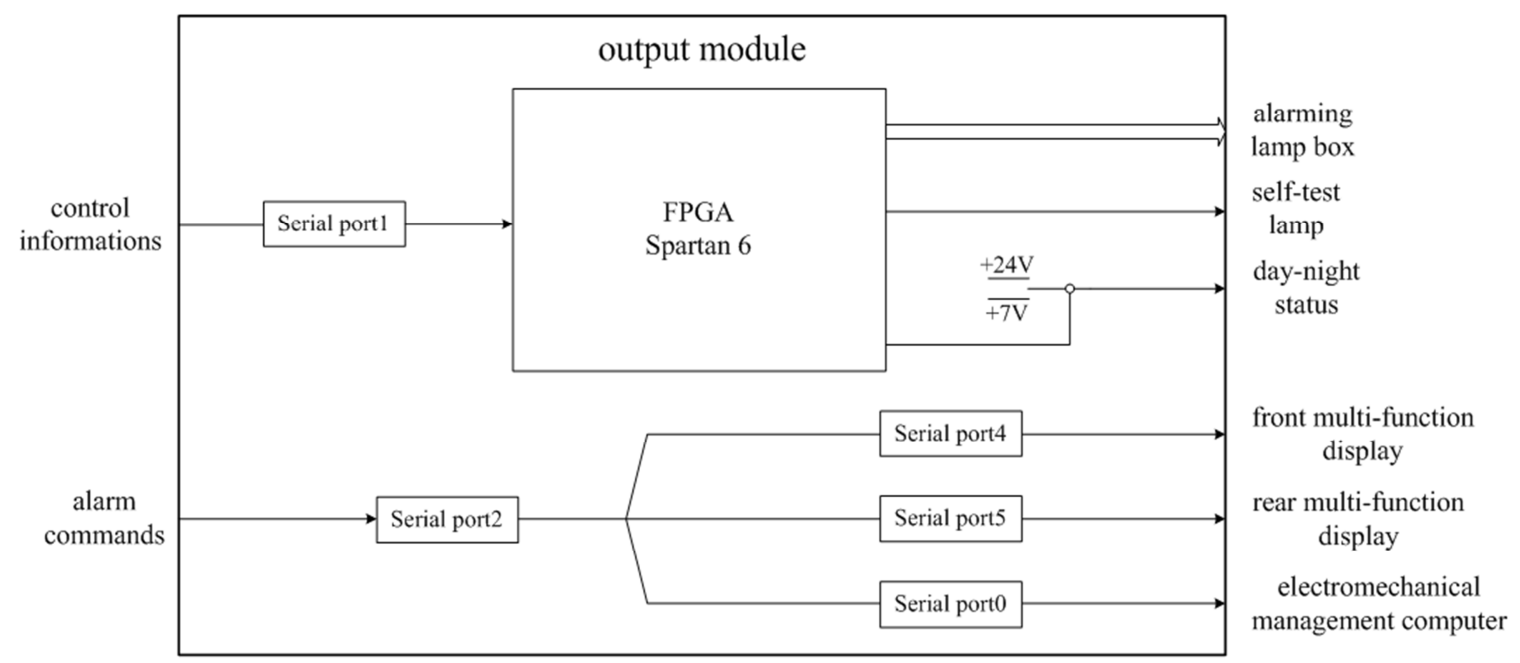

Figure 4. The schematic diagram of output module.

\section{Software Design}

\subsection{Software Flow}

The DSP program of the integrated alarm system needs to complete the following tasks:

(1) Obtain the discrete information from the acquisition module by XINTF bus interface, according to protocol analysis;

(2) Obtain the RS422 data packet from the electromechanical management computer by XINTF bus interface, according to protocol analysis;

(3) Play alarm voice according to the alarm information;

(4) Update the status of the alarming lamp box according to the alarm information, and send them to the output module;

(5) Generate alarm commands according to the alarm information, and send them to the output module.

The program architecture uses time slice circular scheme, each task is a process which sets an identifier. When main program timing cycle arrives, it polls whether the next process is started, otherwise, the previous process keeps running until the end. The advantage of this design is that all 
tasks of the integrated alarm system can be executed within specified time, if a task cannot be returned within a long period of time, it will not preempt the execution cycle of other tasks. As the frequency is high enough, it can be considered that the processor performing all tasks in parallel, the architecture of the software system is shown in Figure 5.

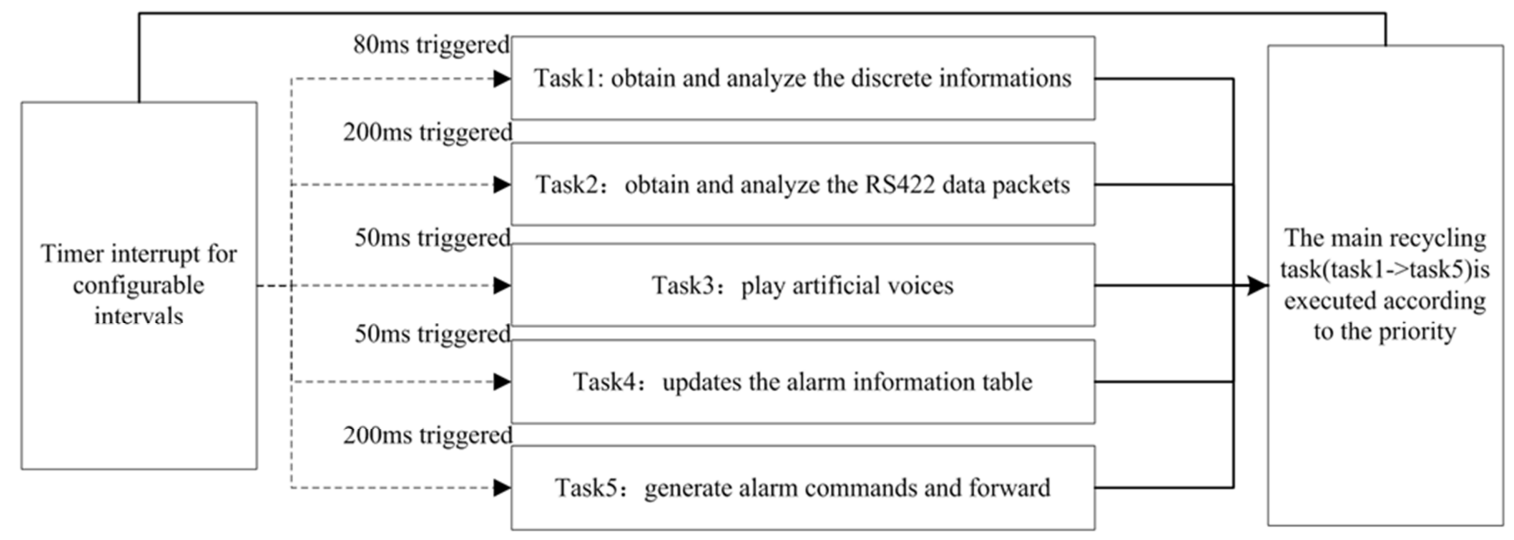

Figure 5. The software architecture diagram of integrated alarm system.
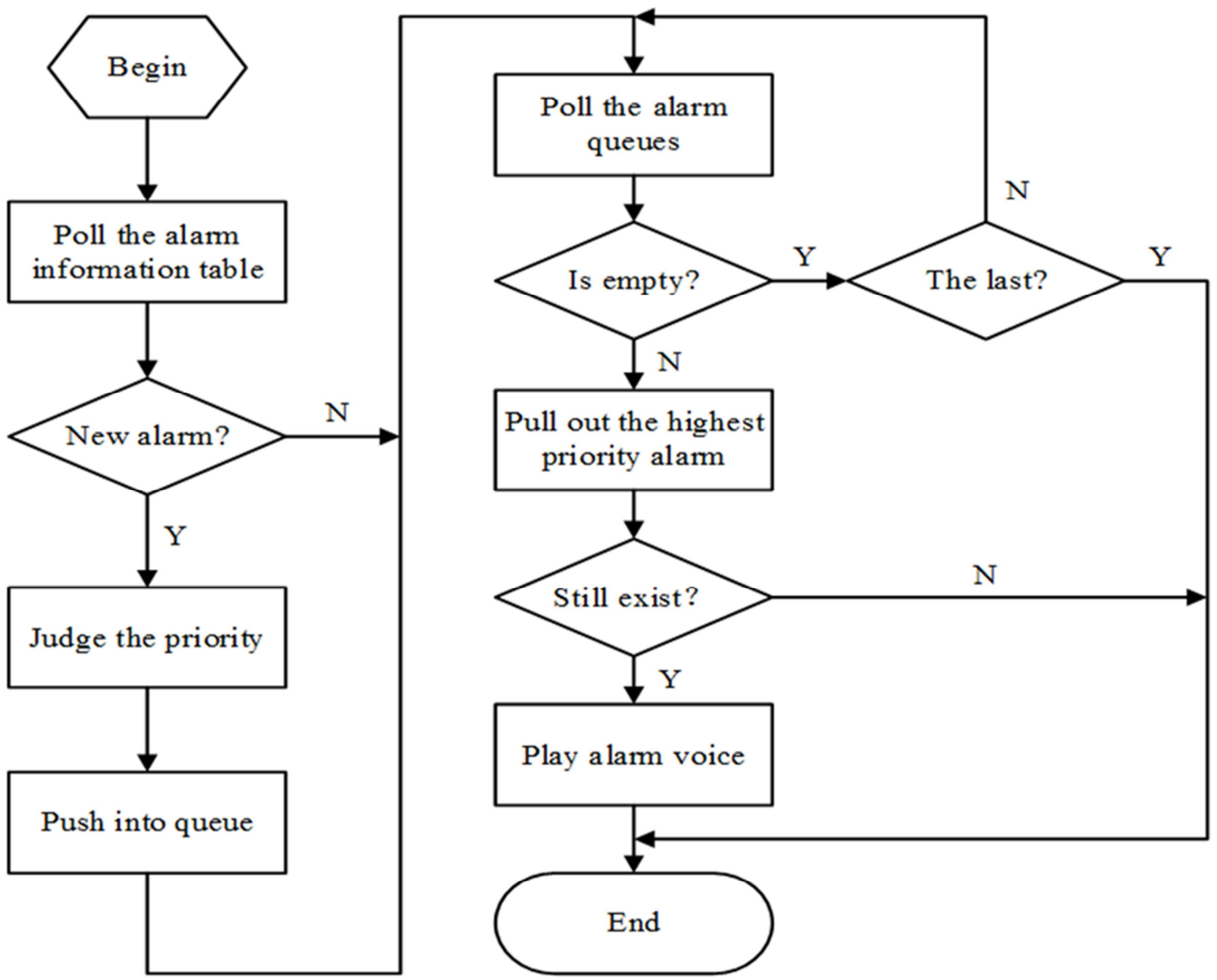

Figure 6. Alarm processing flow chart.

\subsection{Alarm Processing Flow}

According to the urgency of flight safety, the alarm information can be divided into five levels: dangerous level, warning level, attention level, prompt level and status level, the processing priority is successively decreased [9]. Since it takes time to play an artificial voice, if there are multiple alarms in the aircraft, queue-jumping algorithm for the high-priority alarm which is advanced is used for playing artificial voices, the software must first deal with the high-priority voice, and then the low-priority voice, no alarm information is allowed to be missed in this process [10].

In order to achieve the above functions, the software has created five alarm queues in different levels. The DSP obtains the status information of each airborne device, updates the alarm information table, and processes as the following steps [11]. 
1. Poll the alarm information table, if no new alarm is found, go to step 3 [12];

2. If there are new alarms, push into different queues according to the priority [13];

3. Poll all the alarm queues, if queue element is empty, go to step 7;

4. Pull out the highest priority alarm currently, update queues [14];

5. Read the status information, confirm whether the alarm exists, if not, go to step 7 [15];

6. If the alarm still exists, play artificial voices [16];

7. The process ends;

The flow diagram is shown in Figure 6.

\section{Conclusion}

This paper designed an integrated alarm system based on modularization. The system collects device information on the aircraft and notifies the pilot in the form of voice or light by priority. The ground experiment shows that the system is designed reasonable, it can judge the aircraft warning information accurately, play artificial voices hierarchical, and warn the crew of the airplane to deal with problems in time, which greatly improves flight safety.

\section{Acknowledgements}

First of all, I would like to express my highest gratitude to all those who helped me with this paper. Thanks again to my colleagues and partners, and finally to my family, thank you for their continued support and encouragement.

\section{References}

[1] ZHANG Hai-kuo, LU Zhong-hua, LIU Fang, et al. Design and implementation of mass alarm data oriented parallel processing system [J]. Computer Engineering and Design, 2018.

[2] SONG Xiao-gang, ZHAI Zheng-jun, REN Lan-kun. Design of airplane voice-warning system based on ARM [J]. Electronic Design Engineering, 2010, 18 (9): 167-170.

[3] WANG Bo. MP3 Player Design Based on Decoder VS1053 and ADXL345 [J]. Techniques of Automation and Applications, 2014, 33 (7): 43-47.
[4] Zheng W, Wang X. A Design of Music Teleteacher Based on VS1053 Decorder [C]// International Conference on Multimedia Technology. 2010.

[5] SHI Yan-bin, GUO Xin, WANG Hai-long. Design of airplane Sound-warning System Based on Micro-controller [J]. Audio Engineering, 2008, 32 (4): 25-27.

[6] JIA Tao, LIU Zhi-meng. The design and application of the airborne sound alarm system [J]. Journal of Xinyang Agricultural College, 2009, 19 (1): 135-137.

[7] ZENG Jing. The Application of Time Slice Polling Mode in

[8] Building a Real-time System of MCU [J]. Intelligent Computer and Applications, 2010 (2): 15-16.

[9] LIANG xiang-dong. Research and reliablility analysis of XZG-1A integrated warning unit installed on K8E aircraft [D]. Northwestern Polytechnical University, 2002.

[10] Parker L W, Kasemir H W. Airborne Warning Systems for Natural and Aircraft-Initiated Lightning [J]. IEEE Transactions on Electromagnetic Compatibility, 2007, EMC-24 (2): 137-158

[11] Jang G S, Suh S M, Park H Y. Design Strategies of Alarm System for SMART [C] International Conference on Structural Mechanics in Reactor Technology. 2005.

[12] Li X, Zhan Y, Chang L M, et al. Design of comprehensive general support system for airborne inertial platform [C] International Conference on Mechatronic Sciences. IEEE, 2014.

[13] Catros M J Y. Airborne New and Advanced Satellite Techniques and Technologies in a System Integrated Approach $[J]$. Mitteilungen Aus Dem Institut Für Angewandte Mathematik, 2005.

[14] Jiong Z, Bo-Ming X U, Yun-Pu S. Design of Integrated Supervisor and Management Software for Fire Alarm System [J]. Computer Engineering, 2008.

[15] Li X, Chang L M, Zhang L J, et al. Design of Integrated Control and Support Platform for Airborne Digital Image Transmission System [J]. Applied Mechanics and Materials, 2013, 380-384: 2845-2849.

[16] Yu Z. [IEEE Education (ICCSE) - Nanning, China (2009.07.25-2009.07.28)] 2009 4th International Conference on Computer Science \& Education - Study on a new cable guard alarm system based on fuzzy state estimation [J]. 2009: $1867-1872$ 